\title{
The multigate Doppler approach for assessing hemodynamics in a forearm vascular access for hemodialysis purposes
}

\author{
Abigail Swillens \\ Koen Van Canneyt \\ Patrick Segers \\ IBiTech-bioMMeda \\ Ghent University, Ghent, Belgium \\ Email: abigail.swillens@ugent.be
}

\author{
Stefano Ricci \\ Piero Tortoli \\ Information Engineering Department \\ University of Florence, Italy
}

\begin{abstract}
We compared the multigate and single gate pulsed wave Doppler (PWD) approach to assess the hemodynamics in an arteriovenous fistula for hemodialysis purposes. In particular, we evaluated volume flow and wall shear rate measurements based on PWD because: (i) volume flow measurements are typically performed to assess the maturation of the arteriovenous fistula (AVF), with low postoperative fistula flow being a prognostic marker for failure of the AVF; (ii) wall shear stress may play an important role in vascular remodeling processes of the fistula. As in-vivo validation of fistula flow measurements is cumbersome, we performed simulations integrating computational fluid dynamics with an ultrasound (US) simulator. Flow in the arm was calculated, based on a patient-specific model of the arm vasculature pre and post AVF creation. Next, raw ultrasound signals were simulated and processed using a multigate and single gate Doppler approach in the radial artery, both for the preop and postop setting. Results showed that the extreme postop flow conditions (high velocity magnitudes and complex flow profiles) hampered accurate assessment of both wall shear rate and volume flow, while reasonable estimates were obtained preop (lower velocity magnitudes and laminar flow).
\end{abstract}

\section{INTRODUCTION}

Hemodialysis requires a well-functioning vascular access to create a vessel with high blood flow which allows for a repeated cannulation over time. The preferred vascular access is an arteriovenous fistula or AVF, i.e. the surgical connection of an artery and a vein in the arm (e.g. the radial artery and the cephalic vein, cfr. fig.1). This AVF should mature, meaning that the vein should enlarge and the vein wall should enforce to withstand the required flow increase for dialysis. However, non-maturation occurs in 23 to $46 \%$ of the cases [1] despite the extensive preoperative examinations.

The prognosis of maturation of the vein is typically assessed via direct postoperative flow measurements, with too low fistula flow predictive for non-maturation while too high fistula flow may result in distal ischemia or cardiac failure [2]. Nowadays, one of the most widely available flow measuring methods is based on pulse wave Doppler (PWD), typically used to provide info on the centerline velocity. Volume flow can then be obtained by assuming the spatial velocity dis- tribution (e.g. a parabolic, flat or Womersley profile) and estimating the vessel cross-sectional area. While this single gate PWD technique is widely used in daily clinical practice, recent advances in computational power have allowed the real-time use of multigate PWD techniques, simultaneously interrogating a large number of sample volumes along the scanline of interest. For volume flow measurements, multigate techniques are particularly interesting as they allow acquisition of the spatial velocity distribution (in a 1D arterial crosssection), avoiding potential errors made when assuming the velocity profile.

We previously investigated the dependence of volume flow measurements on the assumed spatial velocity profile [3]. This study was based on multiphysics simulations, which granted access to gold standard information on the true velocity field measured by PWD by integrating computational fluid dynamics (CFD) with an ultrasound simulator. As previously demonstrated, this modeling technique allows to simulate synthetic but realistic ultrasound images, based on a known but complex 3D-flow situation [4]. In this study, we will employ the same modeling approach to compare the accuracy of volume flow measurements obtained with multigate and single gate techniques. Flow rates will be derived from synthetic PWD spectra and then compared to the ground truth values known from CFD. Furthermore, we will preliminary evaluate the feasibility of assessing wall shear rate (WSR) in this complex flow setting, as WSR might play an important role in the vascular remodeling processes of the AVF.

\section{Methods}

\section{A. Multiphysics simulations}

We used the Field II software [5], [6] to simulate the radiofrequency (RF) signals originating from the AVF blood flow fields. Field II represents blood as an ensemble of random point scatterers. As such, realistic Doppler spectra were obtained by moving the point scatterers during the simulated scanning procedure according to the AVF flow fields obtained from CFD (cfr. fig.2). 


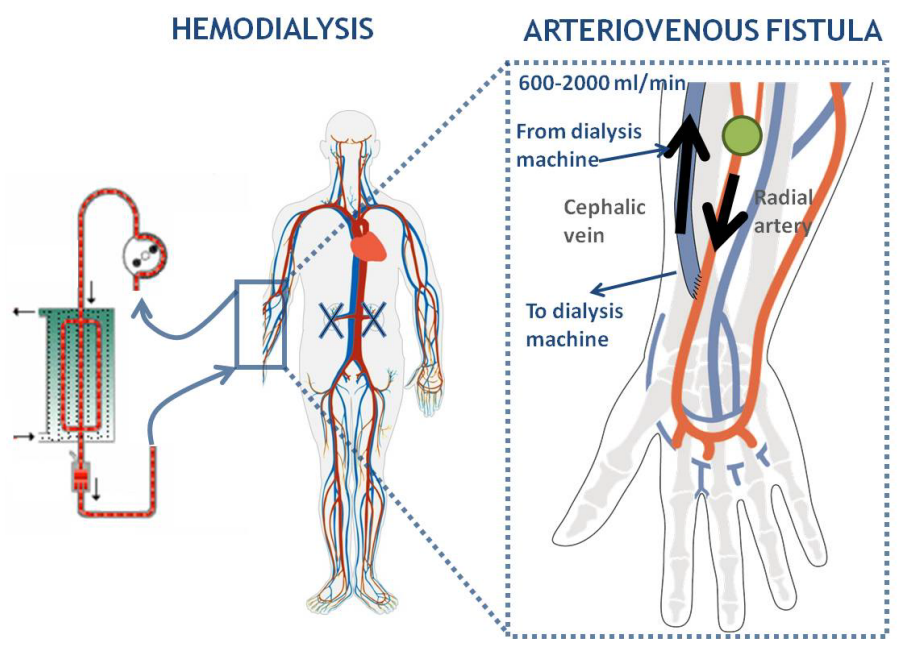

Fig. 1. A radiocephalic fistula for hemodialysis purposes. The green dot in the radial artery illustrates the location where synthetic PWD spectra were generated.

The CFD-simulations were based on 2 sets of highresolution MR angiography images acquired from the same patient (51 years old male), one set preoperatively and one 15 weeks after AVF creation. From these MR data sets, both a preoperative and a postoperative 3D-model were reconstructed using dedicated software (http://www.vmtk.org). The preoperative model consists of the brachial artery, which bifurcates into the ulnar and the radial artery. The postoperative model additionally includes the arteriovenous anastomosis and the distal cephalic vein. A computational mesh of the models was constructed for the CFD-simulations using pyFormex (http://pyformex.org), resulting in a structured hexahedral grid of 1.4 million cells for the preoperative case and 2.2 million cells for the postoperative case. The CFD-simulations further require that appropriate boundary conditions are applied at the in- and outlets of the geometry. As such, a parabolic velocity profile was implemented as inlet boundary condition at the proximal brachial artery, as derived from pre- and postoperative MRI Q-flow acquisition from the selected patient. Traction-free conditions were applied at the outlets. Blood was modelled as an incompressible Newtonian fluid with a density of $1050 \mathrm{~kg} / \mathrm{m}^{3}$ and a viscosity of $3.5 \mathrm{mPa} \cdot \mathrm{s}$. Ansys Fluent 12 (ANSYS inc., Canonsburg, PA, USA) was used to solve the Navier-Stokes equations with a finite volume method. For more background on the ultrasound simulator and the applied multiphysics simulation approach, we refer to [4].
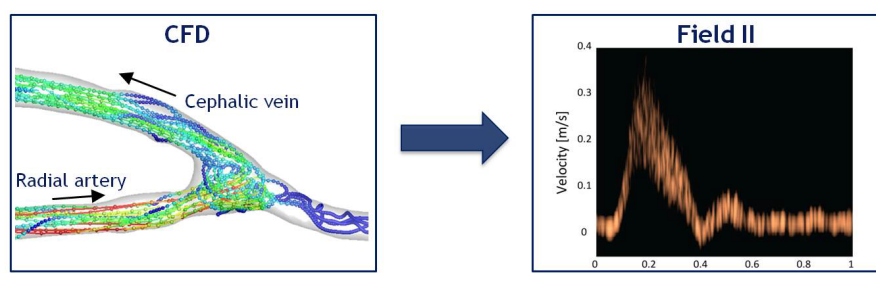

Fig. 2. Illustration of the multiphysics simulation approach.

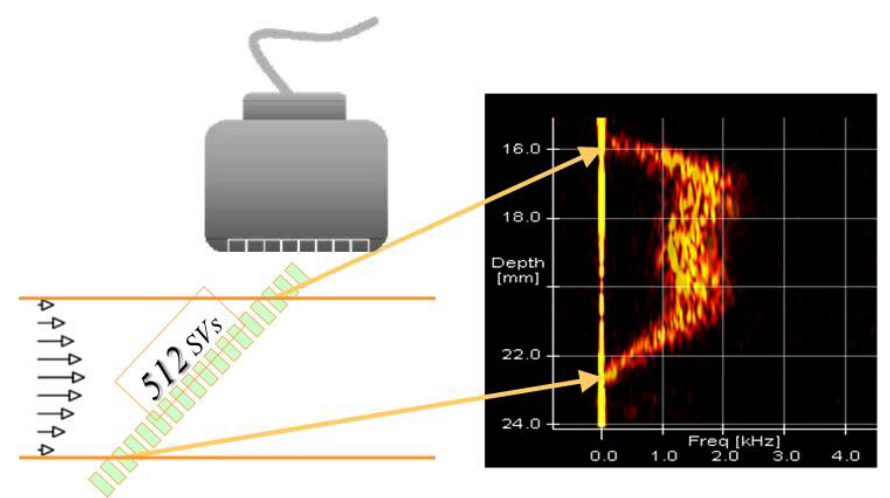

Fig. 3. Illustration of the multigate Doppler approach.

\section{B. Synthetic PWD spectra}

RF-data were simulated in the radial artery, $5 \mathrm{~cm}$ proximal to the anastomosis (cfr. green dot in fig.1), by implementing a realistic linear array transducer for peripheral vascular applications (LA523, Esaote). RF-signals were obtained by firing 12000 and 24000 hanning-windowed sinusoidal pressure pulses during the cardiac cycle, for the preoperative and postoperative case respectively $(\mathrm{f} 0=8 \mathrm{MHz}$, pulse length $=5$ pulse periods). The power spectral density was obtained along the interrogated scanline via Fourier analysis (a windowed 128-point FFT). The power-weighted mean frequency was calculated from these sonograms and converted to velocity $\left(v_{P W D}\right)$ via the classical Doppler equation.

\section{Volume flow calculation based on PWD spectra}

The instantaneous volume flow $\mathrm{Q}(\mathrm{t})$ through a well-defined cross-section A can be calculated as the integration of the spatial velocity profile over the area:

$$
Q(t)=\int_{A}(\vec{v}(t) \cdot \vec{n}) d A=\int_{A} v_{n}(t) d A
$$

with $\vec{v}(t)$ the 3D-velocity vector in a certain point of the cross-section, $\vec{n}$ the normal of the cross-section A and $v_{n}$ the projection of the velocity vector onto the normal. The Doppler velocity $v_{P W D}$ was converted to the angle-corrected velocity $v_{n}(t)$ as: $v_{n}(t)=\frac{v_{P W D}(t)}{\cos (\theta)}$, with $\theta$ the angle between the US beam and the assumed flow direction. In this study, $\theta$ was set to $60^{\circ}$ and $78^{\circ}$ for the preoperative and postoperative case respectively. Evaluation of this surface integral was performed via 2 different strategies:

(i) Single gate Doppler: The velocity is measured at the centre point of the cross-section and is used to derive the spatial velocity profile by assuming the actual flow conditions. The flow formula is then further simplified to: $Q=\beta *$ $v_{n, \max }(t) * A ; \beta$ is a correction factor that accounts for the shape of the velocity profile; $v_{n, \max }$ is the velocity in the centre of the vessel cross-section and assumed the maximal velocity (as is the case for fully symmetrical flow). A parabolic $(\beta=0.5)$ profile was assumed.

(ii) Multigate Doppler: A 1D cross-section of the spatial velocity distribution $v_{n}(t)$ is acquired by simultaneously as- 


\section{Pre-operative}
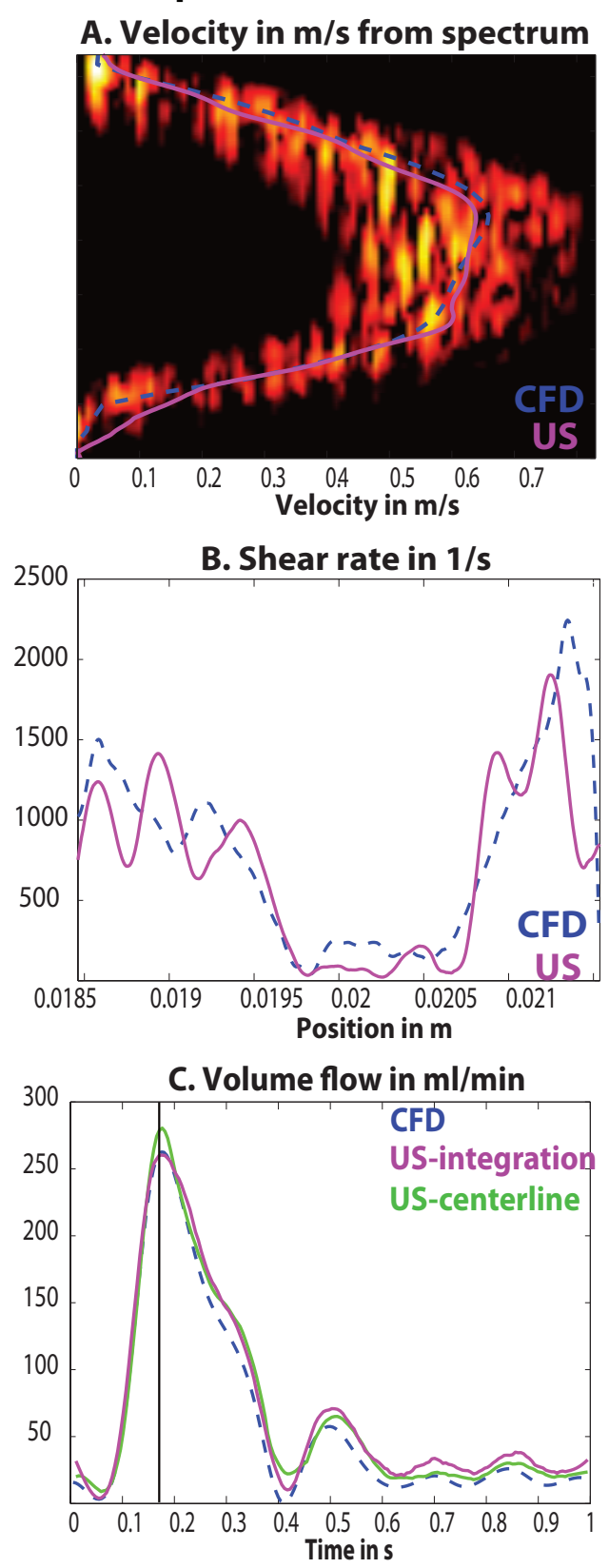

\section{Post-operative}
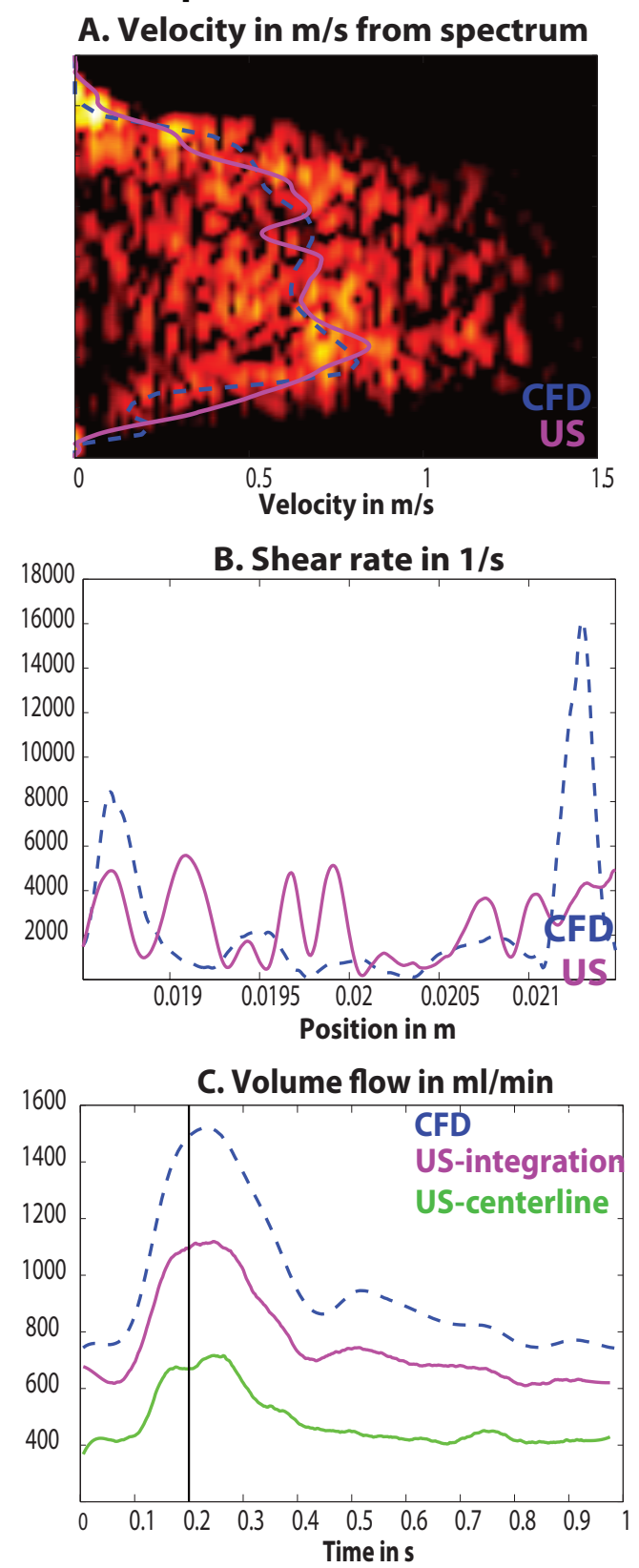

Fig. 4. Preoperative and postoperative results: panels $\mathrm{A}=$ velocity from the multigate spectrum compared to the CFD-reference (blue-dashed), panels $\mathrm{B}=$ the shear rate along the cross-section compared to the CFD-reference (blue-dashed), panels $\mathrm{C}=$ the volume flow during the cardiac cycle as obtained from the single gate (US-centerline) and the multigate (US-integration) technique compared to reference (CFD).

sessing the power density spectrum in an ensemble of sample volumes along the scanline of interest, cfr. fig.3 [7]. The flow $\mathrm{Q}$ is then obtained by estimating the cross-sectional area $\mathrm{A}$ from the vessel diameter and by assuming cylindrical symmetry.

\section{Wall shear rate}

From the multigate acquisition of the spatial velocity distribution, the 1D wall shear rate is derived according to $\tau=\mu \frac{\partial v}{\partial r}$ via numerical differentiation of the velocity profiles [8], [9].
A third order Savitzky-Golay filter was applied to smooth the shear rate data.

\section{RESULTS}

Panels A in figure 4 show the multigate power density spectrum converted to velocity for peak systole (timing indicated in panel $\mathrm{C}$ ) in the preoperative and postoperative case respectively. The pink curve (US) shows the mean velocity curve as compared to the reference CFD-data (blue-dashed). Panels B 
TABLE I

THE ERROR IN \% ON THE TIME-AVERAGE FLOW RATE

\begin{tabular}{|c|c|c|}
\hline Error in $\%$ & Single gate & Multigate \\
\hline Preop & 15 & 19 \\
\hline Postop & -50 & -21 \\
\hline
\end{tabular}

present the corresponding shear rate curves. The volume flow obtained using the single gate (green) and multigate (pink) technique is represented in panels C. Enhanced complex flow is present in the postoperative case as proven by the increased spectral broadening.

In the preoperative case, both the multi- and single gate volume flow data correspond well with the CFD-reference. In the presence of the high postoperative flows, the multigate approach outperforms the single gate technique $(-21 \%$ versus $-50 \%$ ) but both methods seriously underestimate volume flow. The error (in \%) on the simulated volume flow measurements, $\left(Q_{\text {mean }, U S}-Q_{\text {mean }, C F D} / Q_{\text {mean }, C F D}\right)$ is further quantified in table 1 for both measuring strategies and both flow settings.

An accurate measurement of shear rate is challenging, especially for the postoperative setting where the noise in the Doppler velocity profile (fig.4-A) manifests itself in more prominent oscillations in the shear rate curve. Maximum shear rate is underestimated by ultrasound for both flow situations.

\section{DISCUSSION AND CONCLUSION}

In this paper, we have studied PWD-based flow and shear rate measurements in the radial artery of patients with an arterio-venous fistula, both in the pre- and postoperative case. Integration of CFD and Field II simulations allowed validating these hemodynamic assessments towards a ground truth in a highly complex flow setting. Results showed that the extreme postop flow conditions (high velocity magnitudes and complex flow profiles) hampered accurate assessment of both wall shear rate and volume flow, while reasonable estimates were obtained preop (lower velocity magnitudes and laminar flow). The multigate strategy performed similarly as conventional single gate flow techniques in laminar flow conditions but proved superior in the complex flow setting post AVF creation. However, one should note that only one particular imaging setup was investigated, which could be optimized in future work. Other limitations include the lack of surrounding tissue and the absence of a clutter filter during RF signal processing. Furthermore, flow measurements in highly complex flow conditions as present after AVF-creation might benefit from 2D or even 3D flow estimators (e.g. speckle tracking, vector Doppler, transverse oscillation) but their clinical applicability remains to be established.

\section{ACKNOWLEDGMEnTs}

Abigail Swillens is supported by a postdoc fellowship of the Research Foundation Flanders (FWO). This research was partly supported by the European Unions Seventh FrameworkProgramme (FP7/2007-2013) for the Innovative Medicine Initiative under grant agreement number IMI/115006 (the SUMMIT consortium)

\section{REFERENCES}

[1] T.C.Hodges, M. Fillinger, and R. Zwolak, "Longitudinal comparison of dialysis access methods: risk factors for failure," Journal of vascular surgery, vol. 26, pp. 1009-19, 1997.

[2] D. Shemesh, I. Goldin, and D. Berelowitz, "Blood flow volume changes in the maturing arteriovenous access for hemodialysis.," Ultrasound in Medicine and Biology, vol. 33, pp. 727-733, 2007.

[3] K. V. Canneyt, A. Swillens*, L. Lovstakken, L. Antiga, P. Verdonck, and P. Segers, "The accuracy of ultrasound volume flow measurements in the complex flow setting of a forearm vascular access," Journal of Vascular Acces, vol. doi: 10.5301/jva.5000118, 2013.

[4] A. Swillens, L. Lovstakken, J. Kips, H. Torp, and P. Segers, "Ultrasound simulation of complex flow velocity fields based on computational fluid dynamics," IEEE Transactions on Ultrasonics, Ferroelectrics and Frequency Control, vol. 56, no. 3, pp. 546-556, 2009.

[5] J. Jensen, "A new calculation procedure for spatial impulse responses in ultrasound," Journal of the acoustical society of America, vol. 105, pp. 3266-3274, 1999.

[6] J. A. Jensen, "Field: A program for simulating ultrasound systems," Medical and Biological Engineering and Computing, vol. 34, pp. 351352, 1996.

[7] S. Ricci, M. Cinthio, A.R.Ahlgren, and P. Tortoli, "Accuracy and reproducibility of a novel dynamic volume flow measurement method," Ultrasound in medicine and biology, in press: doi:10.1016/j.ultrasmedbio.2013.04.017.

[8] G. Bambi, T. Morganti, S. Ricci, E. Boni, F. Guidi, C. Palombo, and P. Tortoli, "A novel ultrasound instrument for investigation of arterial mechanics," Ultrasonics, vol. 42, no. 4, pp. 731-737, 2004.

[9] P. Tortoli, C. Palombo, L. Ghiadoni, G. Bini, and L. Francalanci, "Simultaneous ultrasound assessment of brachial artery shear stimulus and flow-mediated dilation during reactive hyperemia," Ultrasound in Med. \& Biol., vol. 37, no. 10, pp. 1561-1570, 2011. 